\title{
Des modèles et simulations spatiotemporelles comme " objets intermédiaires " pour mettre en débat l'étalement urbain dans l'île de La Réunion
}

\author{
Participatory spatial models as "boundary objects" to foster \\ dialogue around urban planning and urban sprawl issues on \\ Reunion Island \\ Modelos y simulaciones espacio-temporales como “objetos \\ intermediarios” para discutir la expansión urbana en la Isla de \\ la Reunión
}

\author{
Xavier AUGUSSEAU, Pascal DEGENNE, Danny LO SEEN, Guillaume LESTRELIN \\ et Daniel DAVID
}

Volume 60, numéro 170, septembre 2016

Prospective territoriale participative

Version originale soumise en février 2016. Version révisée reçue en octobre 2016.

URI : https://id.erudit.org/iderudit/1040534ar

DOI : https://doi.org/10.7202/1040534ar

Aller au sommaire du numéro

Éditeur(s)

Département de géographie de l’Université Laval

ISSN

0007-9766 (imprimé)

1708-8968 (numérique)

Découvrir la revue

Citer cet article

AUGUSSEAU, X., DEGENNE, P., LO SEEN, D., LESTRELIN, G. \& DAVID, D. (2016) Des modèles et simulations spatiotemporelles comme " objets intermédiaires " pour mettre en débat l'étalement urbain dans l'île de La Réunion. Cahiers de géographie du Québec, 60(170), 245-264. https://doi.org/10.7202/1040534ar
Résumé de l'article

Un dispositif partenarial mobilisant un outil de simulation paysagère, Ocelet, a été conçu comme support d'un exercice de prospective territoriale sur une intercommunalité de l'île de La Réunion où le mitage agricole représente un enjeu important. La démarche consiste à coconstruire, au sein d'une arène d'une vingtaine d'institutions, des scénarios d'évolution de l'étalement urbain. Pour favoriser ces interactions, des modèles contextualisés sont développés. Dans la démarche, le modèle remplit différentes fonctions au sein de l'arène, de l'initiation à la mise en débat de controverses. Les acteurs du projet s'opposent sur les facteurs concourant à l'étalement urbain et sur les moyens de le réguler. Si ces positions évoluent peu durant le projet, en revanche certaines visions ou certains partis pris ont pu être révisés. La démarche collaborative et le travail de prospective territoriale sont jugés de manière très positive par une large majorité de participants, pour leur rôle de moteurs d'apprentissage collectif. 


\title{
Des modèles et simulations spatiotemporelles comme « objets intermédiaires » pour mettre en débat l'étalement urbain dans l'île de La Réunion
}

\author{
Participatory spatial models as "boundary \\ objects" to foster dialogue around urban \\ planning and urban sprawl issues on \\ Reunion Island
}

Modelos y simulaciones espacio-temporales como "objetos intermediarios" para discutir la expansión urbana en la Isla de la Reunión

\author{
Xavier AUGUSSEAU, Pascal DEGENNE, Danny LO SEEN \\ et Guillaume LESTRELIN \\ UMR TETIS, CIRAD \\ Xavier.Augusseau@cirad.fr \\ Pascal.Degenne@cirad.fr \\ Danny.Lo-Seen@cirad.fr \\ Guillaume.Lestrelin@cirad.fr \\ Daniel DAVID \\ UMR ESPACE DEV, Université de La Réunion \\ Daniel.David.974@gmail.com
}

\begin{abstract}
Résumé
Un dispositif partenarial mobilisant un outil de simulation paysagère, Ocelet, a été conçu comme support d'un exercice de prospective territoriale sur une intercommunalité de l'île de La Réunion où le mitage agricole représente un enjeu important. La démarche consiste à coconstruire, au sein d'une arène d'une vingtaine d'institutions, des scénarios d'évolution de l'étalement urbain. Pour favoriser ces interactions, des modèles contextualisés sont développés. Dans la démarche, le modèle remplit différentes fonctions au sein de l'arène, de l'initiation à la mise en débat de controverses. Les acteurs du projet s'opposent sur les facteurs concourant à l'étalement urbain et sur les moyens de le réguler. Si ces positions évoluent peu durant le projet, en revanche certaines visions ou certains partis pris ont pu être révisés. La démarche collaborative et le travail de prospective territoriale sont jugés de manière très positive par une large majorité de participants, pour leur rôle de moteurs d’apprentissage collectif.
\end{abstract}

\section{Mots-clés}

Étalement urbain, modélisation spatiale, objets intermédiaires, prospective territoriale, recherche collaborative, île de La Réunion.

\begin{abstract}
A collaborative framework using a spatially explicit modeling platform, Ocelet, has been designed as a territorial planning support tool in Reunion Island, where urban sprawl is a major issue. Urban development scenarios and models were constructed collectively by a group of specialists from some twenty institutions. The first group of workshops focused on a range of diverse viewpoints put forward by the different institutions on ways to resolve the problem of uncontrolled urban sprawl. Although this approach did not lead to radical changes in the opinions expressed by the stakeholders involved, a majority of participants were nonetheless positive in their assessment of the broad-based, participatory scenario used for the planning and assessment framework. The vast majority of participants recognized the value of participatory modeling for fostering dialogue and conditions conducive to collective social learning.
\end{abstract}

\section{Keywords}

Urban sprawl, land-use modeling, boundary objects, scenario planning, bridging organization, Reunion Island. 


\section{Resumen}

Un aparato asociativo, que utiliza “Ocelet”, instrumento de simulación paisajístico, se creó como base de un ejercicio de prospección territorial, en una inter comuna de la Isla de la Reunión, donde la proliferación anárquica de construcciones agrícolas representa un problema importante. El proceso consiste en co-construir, en una cancha, una veintena de dispositivos, escenarios de la evolución de la expansión urbana. Modelos contextualizados se construyen con el fin de favorecer tales interacciones. En el proceso, el modelo llena diferentes funciones dentro de la cancha, desde la iniciación hasta el enfrentamiento de controversias. Los actores del proyecto se oponen sobre los factores de la expansión urbana y sobre su reglamentación. Bien que ciertas posiciones cambien poco durante el proyecto, otras visiones o prejuicios han sido revisados. La colaboración y el trabajo de prospección territorial son juzgados positivamente por la mayoría de los participantes, como motor de aprendizaje colectivo.

\section{Palabras claves}

Expansión urbana, modelización espacial, objetos intermediarios, prospección territorial, investigación colaborativa, Isla de la Reunión.

\section{Introduction}

La maîtrise de l'étalement urbain, qui affecte majoritairement le foncier agricole en France (88\% des espaces artificialisés) (CGDD, 2012), est devenu un enjeu prioritaire s'inscrivant désormais dans les préoccupations politiques. En effet, les processus de périurbanisation se traduisent par une forte tension sur l'espace agricole, qui est devenu l'objet de controverses entre acteurs urbains, environnementalistes et intervenants du monde agricole (Bertrand et al., 2006). Les lois du Grenelle de l'environnement ${ }^{1}$ ont récemment donné de nouvelles prérogatives aux intercommunalités pour réguler la consommation par l'urbanisation des espaces agricoles, considérés comme supports d'aménités positives et contribuant au développement durable de ces territoires administratifs. En conséquence, l'aménagement du territoire et l'élaboration de documents comme les schémas de cohérence territoriale (SCOT) ${ }^{2}$ représentent désormais un enjeu de négociation qui intègre de nouveaux acteurs et instances de gouvernance (Jarrige et al., 2006). La diversification des questionnements, sur l'agriculture par exemple, et la pluralité d'acteurs et de perspectives dans les négociations et la prise de décision nécessitent également des innovations sociales et organisationnelles (Bertrand et al., 2005). Ces nouvelles situations de gouvernance "partagée» rendent plus difficiles les choix et la recherche de consensus (Souchard, 2013). Dans des situations complexes où les facteurs d'incertitude sont importants, la prise de décision pour la gestion et l'aménagement des terres devient un véritable défi (McCarthy et Possingham, 2006).

La conduite d'exercices de prospective est souvent combinée avec de la modélisation dans une démarche participative pour explorer des futurs possibles, la modélisation venant en soutien à la construction des scénarios (Walz et al., 2007). La mobilisation de modèles spatiaux pour la prospective permet d'objectiver et de représenter les transformations dans l'espace induites par un scénario (Gourmelon et al., 2012; Voiron-Canicio, 2012).

1 Un ensemble de lois qui visent à mettre en œuvre des engagements en matière d'environnement dans les politiques sectorielles.

2 Document d'urbanisme et de planification d'un projet de territoire réunissant plusieurs communes. 
En outre, le recours à l'information géographique et «l'usage raisonné des représentations spatiales», notamment sous la forme de modèles spatialisés, semblent être une des voies privilégiées pour faciliter la mise en débat de différents points de vue et, potentiellement, dégager des consensus (Lardon et Piveteau, 2005 ; Maurel et al., 2007 ; Batton-Hubert et al., 2008).

Dans une démarche participative, cette catégorie de modèle spatial est ainsi perçue comme un support de négociations plutôt qu'un outil de prise de décision (Verburg et al., 2006). Les démarches de modélisation participative, pour mieux appréhender la complexité des processus à l'œuvre, soulignent le besoin d'impliquer un grand nombre de parties dans l'élaboration de scénarios prospectifs (Wollenberg et al., 2000; Alcamo et al., 2006; Lambin et al., 2006; Kok et Verburg, 2007). L’utilisation de modèles comme objets intermédiaires est revendiquée dans des démarches de médiation qui visent la production de processus d'apprentissage (Antona et al., 2005; Vinck, 2009). Néanmoins, promouvoir des démarches de coconstruction itératives est nécessaire pour assurer une bonne appropriation des modèles et leur mise en débat par les acteurs concernés (Van Ittersum et al., 1998; Volk et al., 2010).

Sur l'île de La Réunion, petit territoire insulaire français situé dans l'océan Indien, l'étalement urbain représente un enjeu important. Un exercice de prospective territoriale, mobilisant un outil de simulation de dynamiques paysagères, a été mené sur le territoire d'une intercommunalité. L'exercice visait à explorer différents scénarios d'évolution de l'occupation du sol dans lesquels l'étalement urbain et ses effets constituent un enjeu central et un point majeur de controverse. Les résultats présentés concernent, d'une part, la construction incrémentielle du modèle urbain et son statut dans la démarche, et d'autre part, l'analyse des interactions entre les acteurs.

\section{Démarche}

À La Réunion, les interactions entre urbanisation, développement agricole et conservation environnementale sont généralement abordées de façon conflictuelle. Ainsi, les compromis nécessaires pour élaborer les documents de planification sont surtout l'expression de rapports de force (Metzger, 2004). Pour aborder ces interactions et tensions de manière plus intégrée, un dispositif de prospective territoriale a été conçu dans le cadre d'une démarche de coconstruction de scénarios qui s’appuie sur des outils de modélisation et de simulation des dynamiques paysagères. Les scénarios, les modèles et les simulations sont mobilisés comme des «objets intermédiaires » (Antona et al., 2005 ; Houet, 2015) permettant d'intégrer des bases de données et des connaissances différentes et de structurer les débats entre les parties prenantes.

L'utilisation de modèles comme objets intermédiaires doit s'inscrire dans une démarche organisant les interactions entre scientifiques et acteurs concernés par le projet. Ces interactions sont considérées nécessaires à un processus d'apprentissage collectif. Les interactions avec les partenaires se déroulent, selon une démarche itérative, au cours de forums - ateliers collectifs et groupes de travail plus restreints durant lesquels le modèle et les simulations sont discutés puis améliorés (figure 1). Ainsi, quatre versions du modèle ont été développées successivement et utilisées lors de cinq réunions ou ateliers qui ont représenté les principaux jalons du projet, lequel s'est déroulé de mars 2012 à décembre 2014. 
Figure 1 La démarche de prospective territoriale

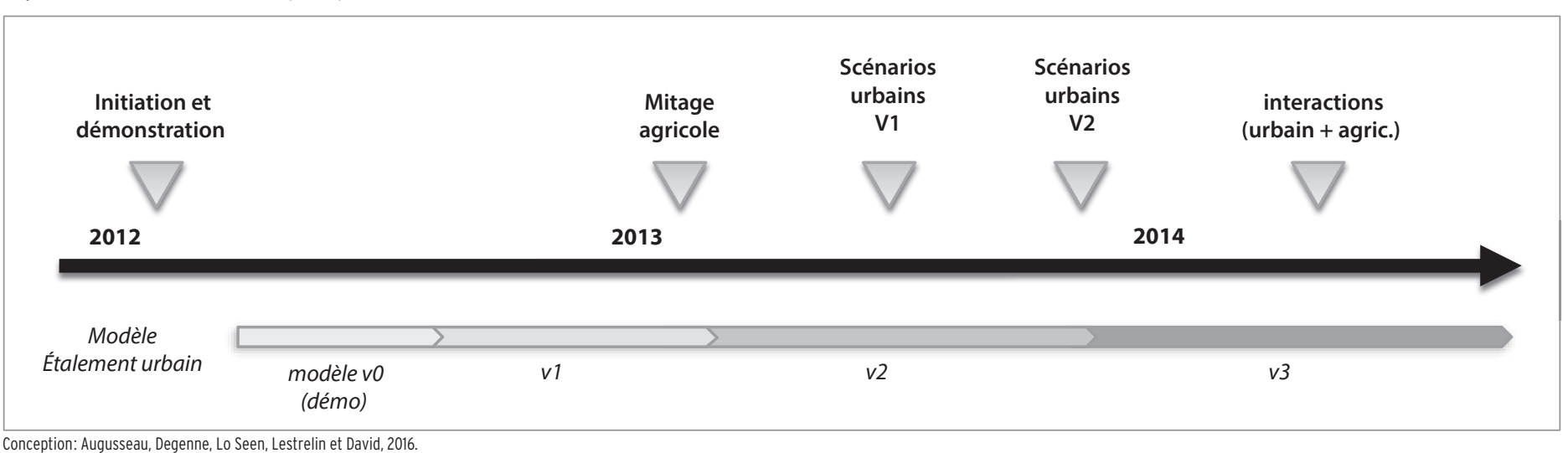


La plateforme de modélisation utilise Ocelet (TETIS, 2016), un langage «métier» de modélisation de dynamiques spatiales et paysagères, qui est basé sur le concept de graphe d'interaction pour exprimer de façon homogène les différents types de relations possibles (spatiales, fonctionnelles, sociales, hiérarchiques) entre les entités du modèle (Degenne et al., 2009; Degenne, 2012). La plateforme permet de restituer les résultats de simulation sous la forme de cartes dynamiques au format KML (Google Earth). En complément et pour répondre aux besoins du projet, des tableaux de bord interactifs présentant des indicateurs agrégés et consultables en ligne ont été développés. En outre, pour favoriser la participation des partenaires dans la démarche et mieux valoriser l'expertise locale, nous avons choisi de développer des modèles contextualisés. Ce type de modèle $a d$ hoc n’a pas de portée générique; il vise à représenter des entités et des processus propres au territoire et familiers à nos partenaires.

Ensuite, dans le cadre du suivi longitudinal de l'exercice de prospective, l'apprentissage a été abordé principalement comme un processus de convergence des perceptions individuelles au sein d'un collectif (Van der Wal et al., 2014). Les données de suivi ont été collectées au moyen de diverses méthodes, incluant une revue de documents stratégiques élaborés par les acteurs du territoire, une série d'entretiens semi-directifs avec ces même acteurs, un travail d'observation participante et une retranscription systématique des discussions lors des différentes réunions du projet, ainsi que des questionnaires d'évaluation distribués à la fin des principaux jalons du projet. Huit thématiques apparaissant comme des points importants de convergence et de divergence entre les acteurs impliqués dans le projet ont été définies lors d'entretiens préalables à l'exercice de prospective (tableau 1). Pour chaque thématique, deux possibilités ont ainsi servi de références pour le codage des données textuelles issues de la transcription des entretiens et ateliers de même que pour le positionnement discursif des acteurs et la cartographie de réseaux d'affiliation/cooccurrence.

Enfin, à l'issue de l'exercice de prospective, nous avons réalisé une analyse du point de vue des acteurs sur les différents éléments de la démarche, les outils mobilisés et les objets intermédiaires produits par le collectif.

Le Territoire de la côte ouest (TCO), un établissement public de coopération intercommunale (EPCI) regroupant cinq communes, a été choisi comme terrain d'expérimentation. Il concentre d'importantes problématiques. Les élus et gestionnaires doivent trouver un équilibre et composer avec une démographie dynamique, des enjeux sociaux importants (70\% des ménages sont éligibles au logement social) et des espaces agricoles qui ont été en partie sanctuarisés (la sole cannière) par des périmètres irrigués. ${ }^{3}$ Le TCO se caractérise également par la présence en aval d'un lagon, dont les ressources sont directement affectées par les activités anthropiques.

À l'est, les zones d'altitude et le cirque de Mafate font partie du cœur du parc national et représentent un pôle touristique très attractif (figure 2). Ainsi, d'importants enjeux environnementaux, notamment sur la qualité de l'eau et les risques liés au ruissellement et à l'érosion, viennent se superposer aux conflits d'usages «historiques» sur le foncier. Enfin, pour mettre en œuvre son projet de territoire - le SCOT - le TCO doit composer avec les politiques sectorielles élaborées aux échelons régionaux et nationaux (ex. : de l'agriculture).

3 Un périmètre réglementaire protège la majorité de la sole cannière et des espaces concernés par un vaste projet d'irrigation. 
Tableau 1 Thématiques définies pour la cartographie des réseaux d'affiliation

\begin{tabular}{|c|c|c|}
\hline Thématique & Possibilité «positive» & Possibilité «négative» \\
\hline Agriculture multifonctionnelle & $\begin{array}{l}\text { Au-delà des questions d'économie rurale, l'agriculture a des fonctions } \\
\text { d'intégration sociale, de lutte contre le chômage, de maintien de services } \\
\text { environnementaux, etc. }\end{array}$ & $\begin{array}{l}\text { L’agriculture est avant tout } \\
\text { une activité économique et de } \\
\text { spécialistes. }\end{array}$ \\
\hline Agriculture et risque nitrate & $\begin{array}{l}\text { L’agriculture contribue de manière significative à réduire la problématique de } \\
\text { pollution par les nitrates. }\end{array}$ & $\begin{array}{l}\text { L'essentiel du problème vient au } \\
\text { contraire de l'assainissement urbain. }\end{array}$ \\
\hline Stratégie agricole et mitage & $\begin{array}{l}\text { Le mitage résulte en partie de défaillances de la part du monde agricole } \\
\text { (ex.: manque d'accompagnement des agriculteurs, etc.). }\end{array}$ & $\begin{array}{l}\text { Le mitage est surtout favorisé par le } \\
\text { jeu politique des élus et le contexte } \\
\text { socioculturel. }\end{array}$ \\
\hline Concertation et coordination & $\begin{array}{l}\text { Le manque de communication entre acteurs limite les réponses aux enjeux } \\
\text { d'aménagement et de développement; il faut travailler en concertation pour faire } \\
\text { émerger des solutions durables. }\end{array}$ & $\begin{array}{l}\text { Certains acteurs ont suffisamment } \\
\text { d'expertise pour gérer eux-mêmes } \\
\text { les problèmes. }\end{array}$ \\
\hline Repenser le modèle agricole des Hauts & $\begin{array}{l}\text { Le modèle actuel est menacé à moyen terme; il faut soutenir la diversification, les } \\
\text { circuits courts, l'agritourisme, etc. pour permettre l'existence de petites structures. }\end{array}$ & RAS, tout va très bien. \\
\hline Repenser le modèle agricole des Bas & $\begin{array}{l}\text { Le modèle actuel est menacé à moyen terme; il faut soutenir la diversification, le } \\
\text { développement du fourrage intensif/irrigué sur les mi-pentes, etc. }\end{array}$ & RAS, tout va très bien. \\
\hline $\begin{array}{l}\text { Intégration économique et territoriale } \\
\text { Hauts-Bas }\end{array}$ & $\begin{array}{l}\text { Il faut accentuer la complémentarité entre les usages et l'économie des Hauts et } \\
\text { des Bas de l'île. }\end{array}$ & RAS, tout va très bien. \\
\hline Régulation du mitage par les besoins & $\begin{array}{l}\text { Le mitage doit être régulé par une meilleure réponse aux besoins en logement; } \\
\text { les objectifs de densification sont difficiles à atteindre, il faut donc pouvoir } \\
\text { urbaniser des zones mitées. }\end{array}$ & $\begin{array}{l}\text { Il faut d'abord protéger les espaces } \\
\text { non urbains, densifier l'urbain } \\
\text { existant et ne pas urbaniser les } \\
\text { zones mitées. }\end{array}$ \\
\hline
\end{tabular}

Conception: Augusseau, Degenne, Lo Seen, Lestrelin et David, 2016 


\section{Tableau 2 Un réseau diversifié d'acteurs}

\begin{tabular}{|c|c|c|c|}
\hline & Milieu urbain & Agriculture & Environnement \\
\hline Services de l'État & $\begin{array}{l}\text { Direction de l'Environnement, de } \\
\text { l'Aménagement et du Logement }\end{array}$ & $\begin{array}{l}\text { Direction de l'Alimentation, de l'Agriculture } \\
\text { et de la Forêt }\end{array}$ & $\begin{array}{l}\text { Direction de l'Environnement, de } \\
\text { l'Aménagement et du Logement }\end{array}$ \\
\hline Collectivités territoriales & $\begin{array}{l}\text { Services techniques des cinq communes; } \\
\text { Conseil régional; } \\
\text { Direction Aménagement Planification et } \\
\text { Prospective du TCO }\end{array}$ & Conseil général & Conseil régional \\
\hline Acteurs parapublics & $\begin{array}{l}\text { Agence régionale des maîtres d'ouvrage } \\
\text { sociaux et aménageurs; } \\
\text { Agence pour l'Observation de } \\
\text { La Réunion, l'Aménagement et l'Habitat }\end{array}$ & $\begin{array}{l}\text { Association Développement rural Réunion; } \\
\text { Chambre d'agriculture; } \\
\text { Société d'aménagement foncier et } \\
\text { d'établissement rural }\end{array}$ & $\begin{array}{l}\text { Parc national de La Réunion; } \\
\text { Réserve naturelle marine de La Réunion; } \\
\text { Association Développement rural Réunion }\end{array}$ \\
\hline Acteurs économiques & & $\begin{array}{l}\text { Tereos; } \\
\text { Syndicat des fabricants de sucre; } \\
\text { Fédération régionale des coopératives agricoles }\end{array}$ & \\
\hline
\end{tabular}

Conception: Augusseau, Degenne, Lo Seen, Lestrelin et David, 2016

$\underline{\tilde{n}}$ 
La démarche s'appuie sur un réseau d'acteurs constitué de 19 institutions de statuts différents, des services de l’État à des associations, et recouvrant des compétences de l'urbain à la gestion de l'environnement (tableau 2). Le profil des personnes participantes diffère non seulement sur les compétences thématiques, mais aussi sur les compétences et expériences dans les usages de l'information spatiale. En complément de ce panel, plusieurs réunions ont été organisées avec les élus du TCO (deux majorités politiques différentes se sont d'ailleurs succédé durant la période du projet), pour amorcer et valider le projet, mettre en débat des scénarios et présenter les résultats.

\section{Figure 2 L'occupation du sol sur le territoire du TCO (2011)}

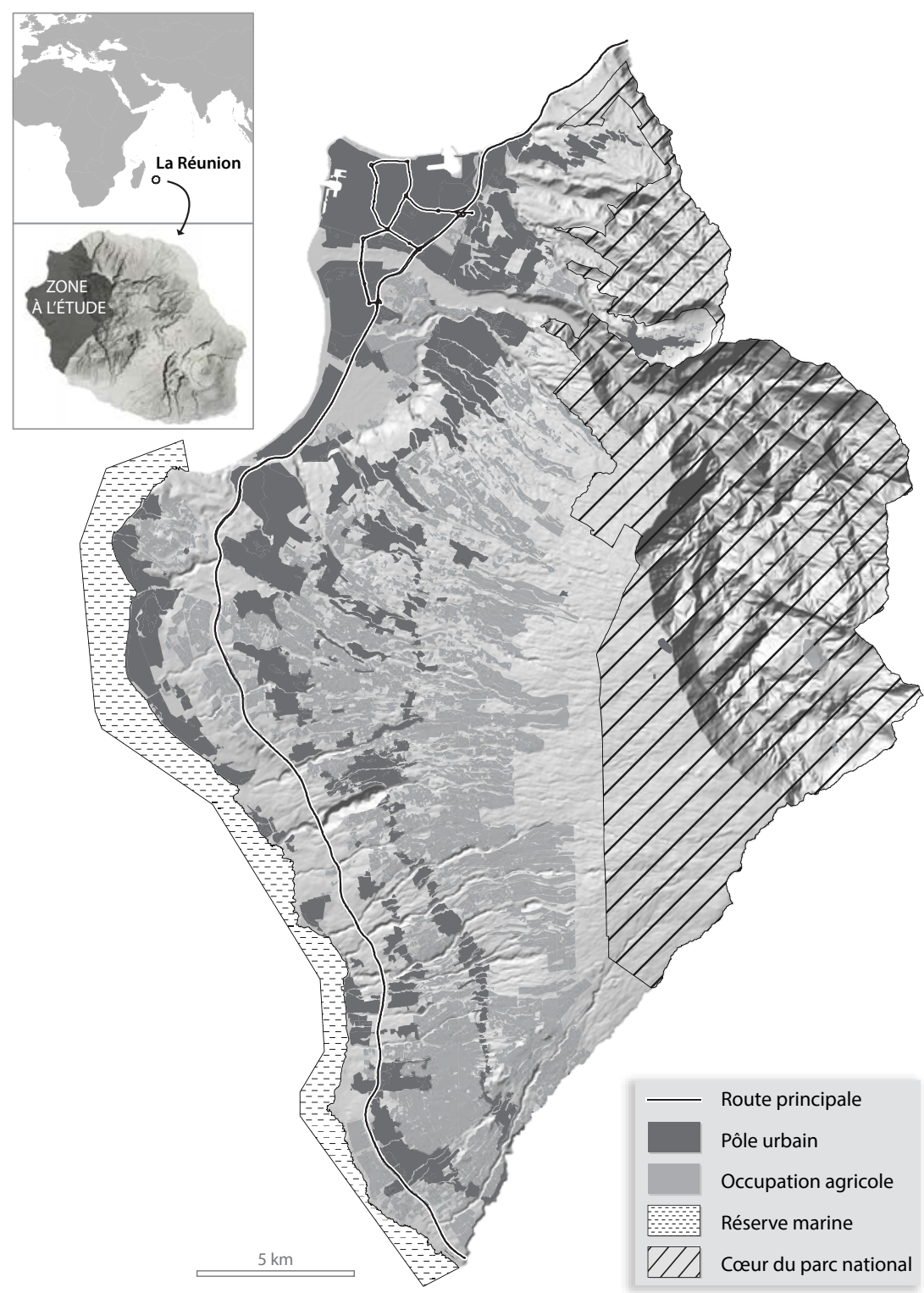




\section{Résultats}

\section{L'évolution du modèle des dynamiques urbaines}

Sur le territoire du TCO, l'urbanisation représente la principale dynamique de changement d'occupation du sol, en raison de la pression démographique et de l'évolution des besoins en logements (décohabitation, etc.). En conséquence, dans cet exercice de prospective, représenter de façon spatialement explicite les processus d'étalement urbain constituait un enjeu important, en particulier pour débattre des conséquences et des effets des dynamiques urbaines sur le territoire.

Un modèle représentant les dynamiques urbaines a ainsi été développé de façon incrémentielle, en quatre versions successives. Cette évolution d'une version à l'autre résulte du processus d'amélioration du modèle, qui se traduit par l'intégration successive de nouveaux processus, en mobilisant les connaissances et l'expertise des acteurs du projet. Néanmoins, dans cette trajectoire, le modèle a également rempli différentes fonctions dans l'exercice de prospective, en particulier dans les interactions au sein du réseau de partenaires.

Une première version du modèle a simulé la construction de logements sur les parcelles cadastrales non bâties des zones urbaines réglementaires (pôles urbains) en fonction d'objectifs de densité (nombre de logements/ha) sur la commune de Saint Pierre, située au sud de l'île (figure 3). La demande de logements était estimée à partir des projections de la Direction de l'Environnement, de l'Aménagement et du Logement (DEAL) (AGORAH, 2008; DEAL, 2012). Des simulations comparaient une dynamique tendancielle, en conservant les densités actuelles, et un scénario qui reprenait les préconisations, en termes de densification, du Schéma d'aménagement régional (CRR, 2011). Le solde de logements, qui n’ont pas pu être bâtis en zone urbaine, est alors «reversé» en zone agricole ou naturelle et représente le mitage. Cette première version a été utilisée comme démonstrateur pour expliciter notre démarche et susciter l'intérêt de l'intercommunalité du TCO. C'est sur cette base que le partenariat a pu être noué et s'est concrétisé par la signature d'une charte de partenariat précisant les objectifs et les modalités de l'exercice de prospective territoriale, à la fin de 2012.

\section{Figure 3 La première version du modèle: un démonstrateur}

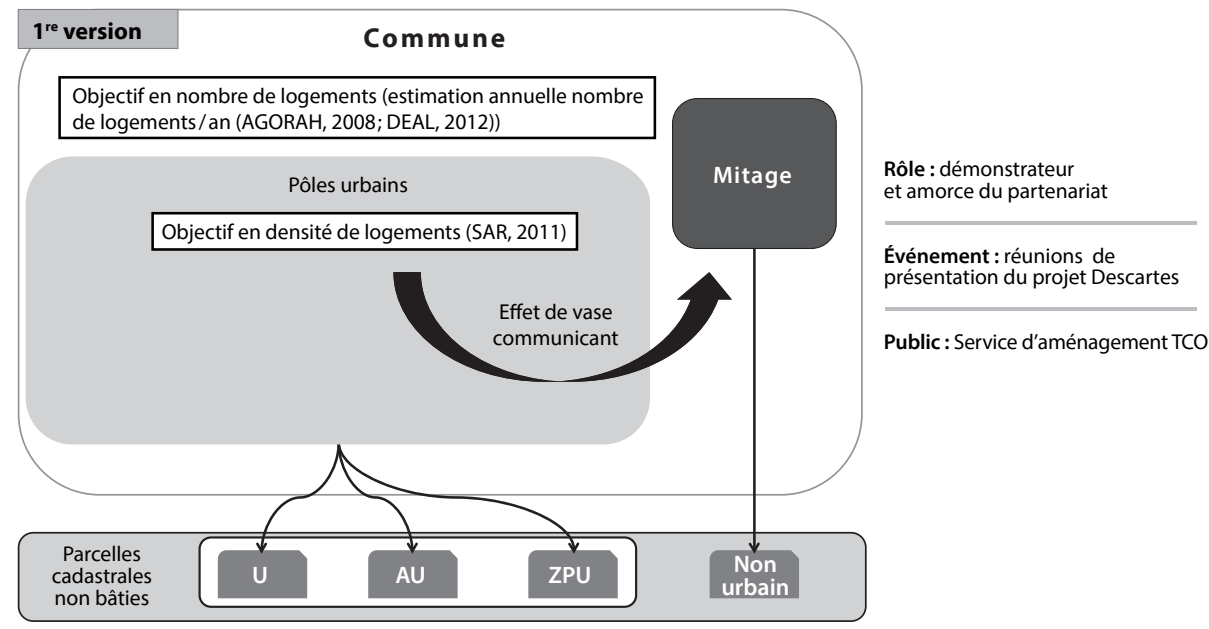

Conception: Augusseau, Degenne, Lo Seen, Lestrelin et David, 2016. 
Le modèle a ensuite été transposé sur le territoire du TCO et le processus de mitage a été affiné (figure 4). À partir d’une analyse rétrospective du mitage sur le TCO, un certain nombre de facteurs ont pu être déterminés et hiérarchisés pour qualifier la propension au mitage des parcelles cadastrales (présence de mitage existant, proximité du réseau routier et de l'urbain, etc.). Cette deuxième version nous a permis, d'une part, d'amorcer le partenariat en présentant notre démarche au futur réseau d'acteurs du projet et, d'autre part, de produire des simulations en soutien à l'atelier sur le mitage agricole, en mars 2013.

Figure 4 La deuxième version du modèle : simulation du mitage

\begin{tabular}{l|l}
\hline $\mathbf{2}^{\mathbf{e}}$ version & \multicolumn{2}{c}{$\begin{array}{l}\text { Rôle : démonstrateur, mise en marche du réseau d'acteurs } \\
\text { et soutien d'atelier }\end{array}$} \\
\hline $\begin{array}{l}\text { - Le modèle est implanté sur le TCO } \\
\text { Différents facteurs ont été introduits et hiérarchisés } \\
\text { pour simuler le mitage des parcelles }\end{array}$ & $\begin{array}{l}\text { Événement : atelier sur le mitage agricole } \\
\text { Public : réseau de partenaires }\end{array}$ \\
\multicolumn{1}{c|}{ Conception: Augusseau, Degenne, Lo Seen, Lestrelin et David, 2016. }
\end{tabular}

La troisième version représente une évolution majeure, puisque l'objectif est de représenter la première itération des scénarios qui ont été mis en débat. Ces premiers scénarios ont été élaborés avec l'équipe de la Direction de l'aménagement du TCO. De nouvelles entités et de nouveaux processus sont introduits dans le modèle. Les processus de construction de logements prennent en compte les projets de planification qui sont intégrés dans le Plan local d'habitat (PLH). En outre, nous avons distingué les différents types de logement qui relèvent de la planification (logement social et aménageurs privés) et le marché libre (résidentiel, etc.) (figure 5). La présentation des scénarios et des simulations ainsi que les débats associés ont permis de déterminer des éléments à prendre en compte pour améliorer et affiner le modèle. Ainsi, il y a eu consensus entre les participants pour que les scénarios prennent mieux en compte la question de la densification urbaine, de même que les conditions de mise en œuvre de l'aménagement urbain (accès aux différents financements publics, coûts et maîtrise publique du foncier au sein de l'espace urbain). En outre, le modèle devrait pouvoir représenter les effets des scénarios sur la production de différents types de logement et leur répartition spatiale.

Enfin, dans la dernière version, des adaptations ont été introduites pour mieux représenter la deuxième variante des scénarios. En particulier, une variable «température urbaine», essentiellement basée sur le prix du foncier, a été calculée pour chaque parcelle cadastrale urbaine afin d'affiner le processus d'étalement urbain. Le modèle a été adapté pour que le processus de vases communicants (planification vers libre diffus ou mitage) puisse être paramétré pour tester différentes hypothèses de transfert (figure 6). Enfin, en complément, des cartes dynamiques (figure 7) des indicateurs sur les types de logement et par différents niveaux d'organisation territoriale (pôle urbain, commune et TCO) ont été réalisées et représentées sous la forme de tableaux de bord interactifs. Durant le séminaire final, le modèle a été notamment utilisé pour explorer des controverses comme les effets des différents scénarios de politique urbaine sur le mitage, qui ont fait débat au sein de l'arène depuis le début du projet (le mitage est-il une conséquence du déficit de logement?). 
Figure 5 Le troisième modèle représente les premiers scénarios

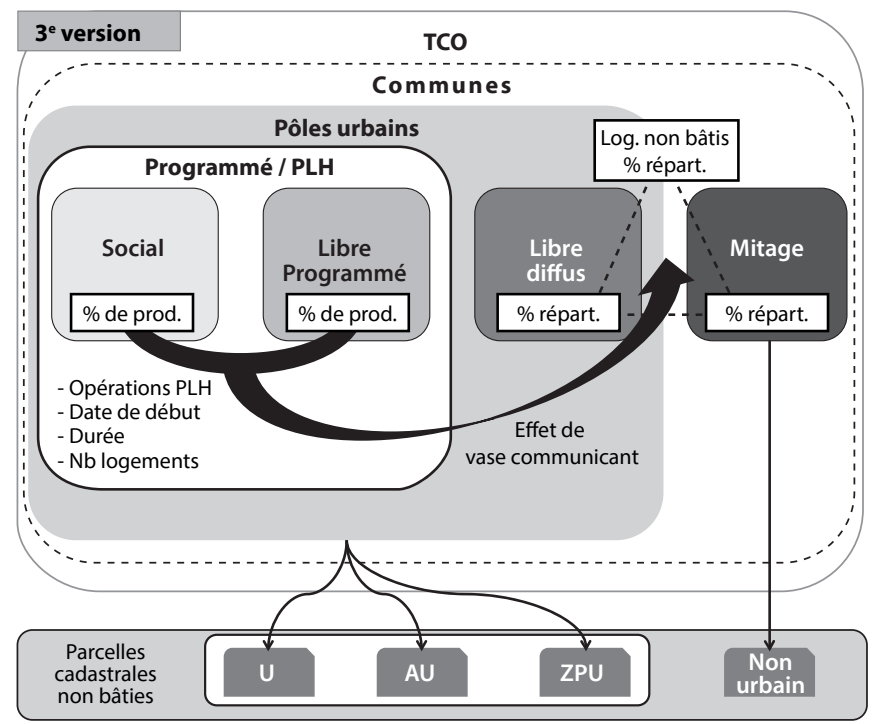

Rôle : représentation des scénarios

Evénement : atelier de prospective

Public : réseau de partenaires

Conception: Augusseau, Degenne, Lo Seen, Lestrelin et David, 2016.

Figure 6 Le quatrième modèle

\begin{tabular}{|l|}
\hline $4^{\mathbf{e}}$ version \\
\hline - Introduction de la temporalité dans la \\
réalisation des opérations d'aménagements du PLH \\
- Intégration d'une «température urbaine " \\
(prix du foncier, composantes espace urbain, éloignement de l'urbain, pente...) \\
pour répartir le logement "libre diffus" \\
- Paramétrisation plus fine des flux de types de logement en fonction \\
des succès ou échecs des opérations programmées libres ou sociales \\
(le vase communicant)
\end{tabular}

Rôle : réprésentation des scénarios et mise en débat de controverses

\section{Événement : séminaire final}

Public : réseau de partenaires et membres externes invités (scientifiques, etc.)

Conception: Augusseau, Degenne, Lo Seen, Lestrelin et David, 2016.

Figure 7 Extrait des sorties cartographiques selon la version du modèle urbain

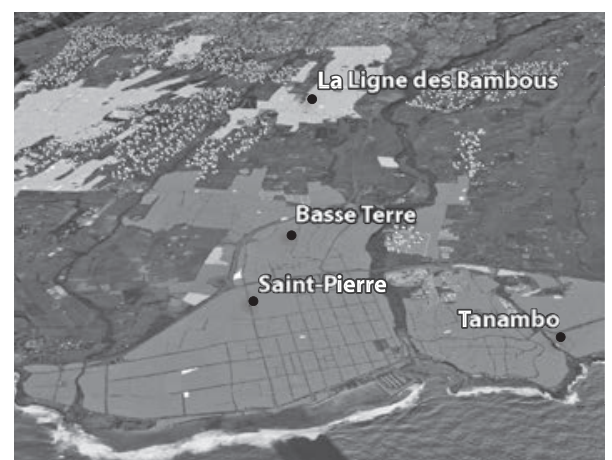

La première version, sur la commune de Saint Pierre Conception: Augusseau, Degenne, Lo Seen, Lestrelin et David, 2016.

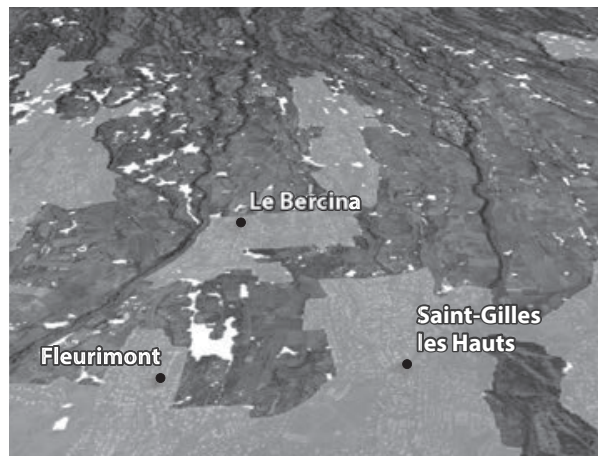

La version finale, sur le territoire du TCO 


\section{Les interactions au sein de l'arène}

\section{Un réseau d'acteurs « clivé »}

L’analyse des discours permet de qualifier, de manière relativement détaillée et sur une base semi-quantitative, le positionnement relatif, les coalitions et oppositions discursives des acteurs autour d'antagonismes et de points de convergence particuliers qui structurent le débat sur l'aménagement du territoire du TCO.

La représentation en réseau valué et signé du positionnement relatif des acteurs au cours des 15 mois de suivi montre que le réseau d'acteurs se caractérise par des positions assez contrastées (figure 8). S'il n'existe pas d'opposition franche entre acteurs partageant les mêmes champs d'expertise (urbaine, agricole, environnementale et rurale), il n'y a pas toujours une convergence de positionnement. Le suivi du positionnement discursif des acteurs autour de huit thématiques différentes a notamment montré une opposition persistante entre les mondes urbain et agricole sur quelques sujets-clés. Par exemple, la nécessité de faire des compromis entre espace agricole et urbain et, notamment, de permettre l'urbanisation des zones mitées (vision promue par les acteurs du développement urbain mais réfutée par le monde agricole) a été longuement discutée. Les acteurs urbains, ceux des collectivités locales en particulier, se démarquent également par une forte cohésion dans leur positionnement, en comparaison aux acteurs du monde agricole, qui semblent s'affirmer essentiellement dans l'opposition au monde urbain.

Figure 8 Réseau de cooccurrence des discours par soustraction des positionnements conflictuels
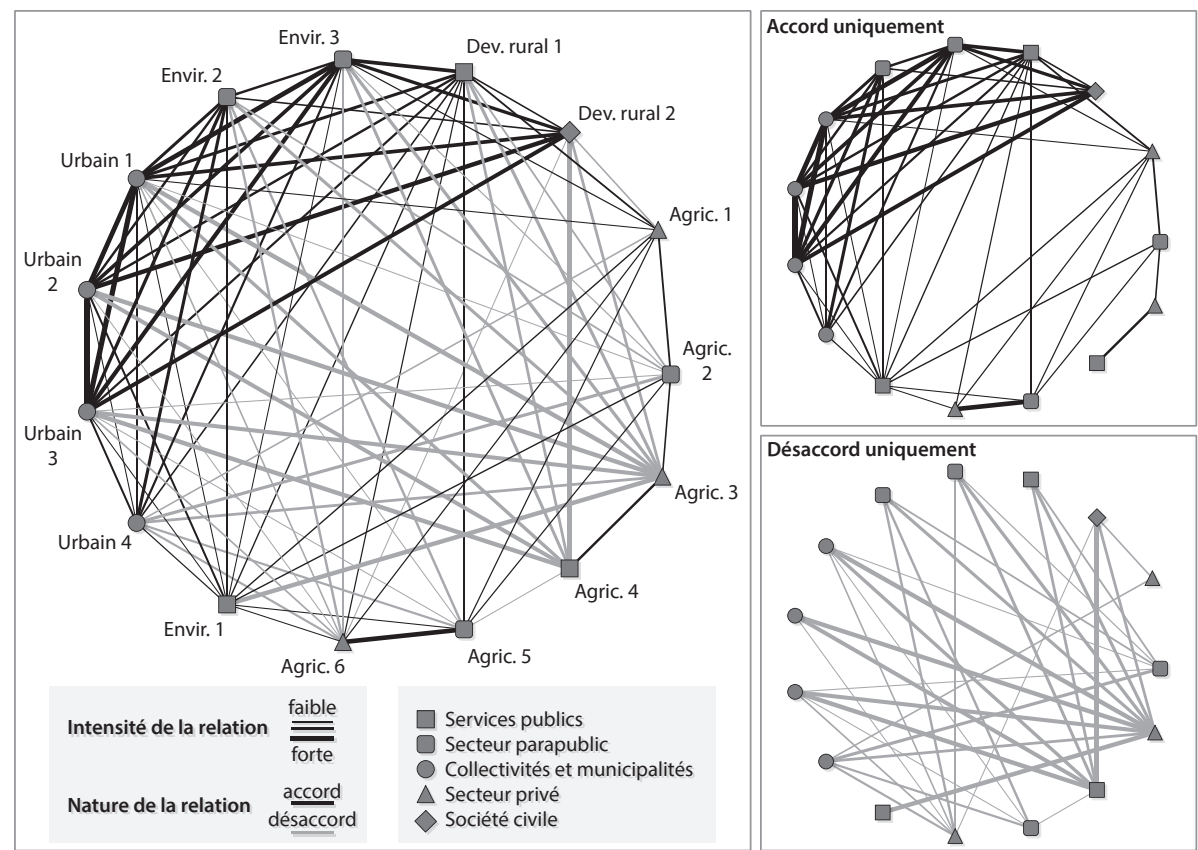

Conception: Augusseau, Degenne, Lo Seen, Lestrelin et David, 2016. 
Si ces positionnements et clivages discursifs n'ont quasiment pas évolué au cours du projet, dans les faits, certains acteurs ont toutefois accepté de considérer et d'intégrer des perspectives promues par d'autres. Lors des débats sur les scénarios, par exemple, la vision des acteurs agricoles, très réglementaire et inspirée par les documents de planification territoriale (ex.: le Schéma d'aménagement régional qui promeut la densification urbaine), s'est opposée à la vision de certains acteurs urbains, plus pragmatique et empreinte d'expérience des difficultés rencontrées lors de la réalisation de projets d'urbanisation (retards liés à des problèmes de maîtrise foncière, de financement, etc.). Débattue au cours de différents ateliers et intégrée par les acteurs du monde agricole, c'est au final cette dernière vision qui a nourri l'essentiel du travail de coconstruction de scénarios prospectifs. Les scénarios se sont ainsi appuyés sur les projections du Plan local de l'habitat intégrant, par exemple, un effet «retard» dans les projets d'aménagement urbain.

\section{L'évolution du point de vue des acteurs sur les outils et la démarche du projet}

Lors de l'atelier de démarrage du projet, la réaction des participants aux propositions de l'équipe de recherche d'élaborer des activités de prospective territoriale a été très variable: certains ont montré un bonne compréhension et un intérêt marqué pour une démarche de modélisation spatiale et d'exploration de scénarios ; d'autres étaient moins convaincus ou n'avaient pas une vision claire de la démarche; enfin, certains qui, tout en étant intéressés par les outils de modélisation spatiale, étaient plutôt rétifs à son utilisation dans un cadre collaboratif ou à visée intégrative. Néanmoins, malgré cette diversité de points de vue, le projet a réussi à mobiliser les représentants de treize à seize institutions dans les cinq ateliers qui ont jalonné les deux années du projet, sans compter les multiples interactions (réunions, entretiens, échange de données, etc.) qui ont été organisées avec l'équipe scientifique.

Cette dynamique partenariale s'est confirmée à l'issue du séminaire final. Les résultats de l'enquête réalisée auprès des participants et les commentaires associés indiquent que la démarche collaborative et le travail de prospective territoriale sont jugés de manière très positive par une large majorité de participants (tableau 3). Ils sont appréciés essentiellement pour leur rôle de moteurs d'apprentissage collectif (c'est-à-dire prise en compte de différents points de vue, meilleure connaissance des problématiques d'autres secteurs d'activité et, dans une moindre mesure, meilleure connaissance des acteurs du territoire). L'apport de la modélisation et de la simulation spatiale est également perçu de manière positive, même si tous les participants n’ont pas les mêmes compétences et expériences pour appréhender la complexité croissante des modèles issus de la démarche itérative. D’une part, tous n’ont pas la même expertise thématique (par exemple, sur l'urbain) et, d'autre part, certains n'ont pas d'expérience de l'usage de l'information spatiale. 
Tableau 3 Évaluation finale du projet et de la démarche par les participants $(n=13)$

\begin{tabular}{l|c|c|c|c|c}
\hline & $\begin{array}{c}\text { Très } \\
\text { négatif }\end{array}$ & $\begin{array}{c}\text { Plutôt } \\
\text { négatif }\end{array}$ & $\begin{array}{c}\text { Neutre/ } \\
\text { Moyen }\end{array}$ & $\begin{array}{c}\text { Plutôt } \\
\text { positif }\end{array}$ & $\begin{array}{c}\text { Très } \\
\text { positif }\end{array}$ \\
\hline $\begin{array}{l}\text { Prise en compte des points de vue } \\
\text { et idées des participants }\end{array}$ & 0 & 0 & 3 & 7 & 2 \\
\hline $\begin{array}{l}\text { Compréhension du } \\
\text { fonctionnement des modèles }\end{array}$ & 0 & 0 & 5 & 6 & 2 \\
\hline Pertinence des indicateurs et cartes & 0 & 1 & 1 & 7 & 4 \\
\hline $\begin{array}{l}\text { Découverte d'acteurs pas ou peu } \\
\text { connus }\end{array}$ & 1 & 3 & 4 & 2 & 3 \\
\hline $\begin{array}{l}\text { Découverte des problématiques } \\
\text { d'autres secteurs }\end{array}$ & 0 & 0 & 7 & 4 & 2 \\
\hline $\begin{array}{l}\text { Changement de point de vue sur } \\
\text { des questions spécifiques }\end{array}$ & 1 & 5 & 2 & 3 & 1 \\
\hline $\begin{array}{l}\text { Utilité de la démarche prospective } \\
\text { et scénarios }\end{array}$ & 0 & 0 & 2 & 4 & 7 \\
\hline $\begin{array}{l}\text { Utilité de la modélisation et } \\
\text { simulation }\end{array}$ & 0 & 1 & 3 & 3 & 5 \\
\hline Conception: Augusseau, Degenne, Lo Seen, Lestrelin et David, 2016 & & & & \\
\hline
\end{tabular}

Concernant l'usage de modèles, ce sont surtout les représentations graphiques issues des simulations, sous la forme de cartes dynamiques, qui ont fait l'objet de débats récurrents pendant le projet. Lors de la phase d'initiation et de démonstration, les cartes produites par les modèles démonstrateurs ont eu un effet d'attraction indéniable et se sont révélées utiles pour engager le dialogue. Cependant, les cartes des simulations des différents scénarios ont fait l'objet de discussions, en particulier sur le risque d'une mauvaise interprétation de ces cartes, notamment par des élus ou des acteurs extérieurs à l'arène du projet. Une partie de nos partenaires considérait que la granularité des cartes et la vision très réaliste en 3D pouvaient dénaturer la dimension exploratoire des simulations en donnant un caractère prédictif aux simulations. Cette réaction montre que pour un enjeu aussi sensible que le foncier qui oppose, dans notre cas, acteurs urbains et agricoles, le type de représentation des sorties du modèle n'est pas neutre. Le réalisme ne favorise pas nécessaire l'exploration de différents futurs possibles.

\section{La vision des acteurs sur l'usage et la finalité des outils mobilisés}

Les activités de suivi de l'exercice de prospective ont aussi été l'occasion de recenser les différentes perspectives des acteurs sur le travail de modélisation/simulation per se et la façon de le présenter. D’une manière générale, le positionnement des participants sur la finalité des outils oscille entre outils de prospective pour aider à la prise de décision et outils de communication mobilisables pour influencer les décideurs. Du point de vue de l'application prospective, l'accent peut également être mis sur le rôle des modèles/simulations, soit comme déclencheurs et médiateurs de débats entre acteurs, soit comme outils d'expertise pour l'évaluation ex-ante. Selon les différentes perspectives, les principaux éléments de la démarche comme l'effort de modélisation requis, la représentation des simulations et le type d'indicateurs utilisés vont se décliner différemment. Le tableau 4 propose une représentation synthétique des différentes visions recensées. 


\section{Tableau 4 Perspectives des acteurs sur le travail de modélisation et de simulation}

\begin{tabular}{|c|c|c|c|}
\hline $\begin{array}{l}\text { Modèles et simulations } \\
\text { comme... }\end{array}$ & Outils de communication & \multicolumn{2}{|c|}{ Outils de prospective } \\
\hline \multirow[t]{2}{*}{ Finalité/objectifs } & \multirow[t]{2}{*}{ Influencer la prise de décision } & \multicolumn{2}{|l|}{ Aider à la prise de décision } \\
\hline & & Faciliter le débat entre acteurs & Permettre l'évaluation ex-ante \\
\hline Effort de modélisation & $\begin{array}{l}\text { Faible, l'essentiel est dans le } \\
\text { message à transmettre }\end{array}$ & $\begin{array}{l}\text { Modéré, on cherche un équilibre entre } \\
\text { réalisme des modèles et dynamisme des } \\
\text { débats }\end{array}$ & $\begin{array}{l}\text { Fort, il faut décrire le champ des possibles avec } \\
\text { précision }\end{array}$ \\
\hline $\begin{array}{l}\text { Représentation des } \\
\text { simulations }\end{array}$ & Très réaliste, ex. : $3 D$ paysagère & $\begin{array}{l}\text { Flexible et diversifiée, adaptée à la } \\
\text { diversité du public cible }\end{array}$ & Scientifique, ex. : cartographie dynamique d'impact \\
\hline Types d'indicateurs & Basiques, principalement visuels & \multicolumn{2}{|l|}{$\begin{array}{l}\text { Potentiellement complexes et variés } \\
\text { (qualitatifs-quantitatifs...) }\end{array}$} \\
\hline
\end{tabular}


Ainsi, lors de nos différentes réunions bilatérales, les représentants de la filière sucrière ont généralement insisté sur la nécessité de limiter l'effort de modélisation à une cartographie du risque de mitage agricole, sans différencier les productions agricoles présentes sur le territoire et sans aborder la question des futurs possibles de l'agriculture sur le TCO, considérant que ces dernières questions sont avant tout liées à la mise en œuvre de politiques urbaines économes en foncier. La finalité d'un tel exercice de modélisation / simulation est alors d'étayer un argumentaire sur la nécessité de soutenir et intégrer l'agriculture dans les projets de territoire. D’autres acteurs, notamment des élus du TCO, des représentants de la DEAL ou de la Réserve marine, ont eu plutôt tendance à considérer la modélisation et la simulation comme des outils d'expertise pour l'évaluation ex ante permettant, par exemple, de sélectionner des zones adaptées pour la construction de retenues collinaires dans les Hauts du TCO ou de prédire les effets d'événements pluvieux sur le ruissellement et le transfert de sédiments de l'amont vers l'aval. Au final, seuls les services techniques du TCO ont, très tôt, adopté une perspective similaire à celle de l'équipe scientifique du projet, considérant les modèles, simulations et scénarios comme des moyens privilégiés de faciliter le dialogue et structurer les débats entre acteurs de l'aménagement du territoire et, en particulier, entre les acteurs du développement urbain et le monde agricole.

\section{Discussion}

Un des enjeux de notre démarche était de lever les contraintes liées à la fragmentation des données et des connaissances sur la thématique de l'étalement urbain. Si la modélisation représente, en soi, un exercice d'intégration de connaissances, l'expérience de ce projet montre que c'est finalement l'ensemble de la démarche qui participe à cette intégration (Stenseke, 2009; Van Berkel et Verburg, 2012). En effet, les phases de coconstruction de scénarios et de développement de modèles servent à formaliser tout le travail, en amont, d'exploration puis de mise en forme de données, des connaissances et de l'expertise sur les dynamiques territoriales et la mise en œuvre des politiques publiques. Les modèles sont ainsi conçus comme des objets d'intégration des connaissances des différents acteurs du TCO. L'expertise de ces acteurs a permis de mobiliser des connaissances très fines pour réaliser des modèles dans lesquels les processus intégrés sont, de fait, très dépendants du territoire. La plateforme Ocelet, qui représente de façon homogène les différentes formes de relation (spatiales, hiérarchiques, sociales, etc.) entre les entités d'un modèle, facilite ce processus d’intégration.

Le développement d'un modèle démonstrateur sur l'étalement urbain, la présentation de simulations issues de ce modèle et le choix du mitage comme thématique principale pour les premières activités du projet ont constitué des éléments cruciaux pour la structuration d'une arène de travail collaboratif. Reconnue par les participants à l'atelier de démarrage du projet comme une préoccupation importante pour les différents acteurs de l'aménagement du territoire, la problématique du mitage représente ainsi un «objet clivant» intéressant, à la fois sujet à controverse entre partisans de différents discours (sur les facteurs et mécanismes sous-jacents, les stratégies de régulation, etc.) et dynamique territoriale émergente qui réexamine les politiques urbaines et agricoles à l'œuvre, ainsi que leur intégration. Pour l'équipe scientifique, mettre l'accent sur cette problématique, c'était donc à la fois s'assurer d'attirer l'attention d'un grand nombre d'acteurs et se donner les moyens de favoriser, dès le démarrage du projet, les questions transversales et une démarche intégrative.

En parallèle, le recours à un langage métier comme Ocelet, les travaux de modélisation participative et leur rendu sous forme de cartes dynamiques ont permis de faire évoluer rapidement le modèle d'étalement urbain vers une représentation plus «pertinente, crédible 
et légitime» (Cash et al., 2003: 8086) des dynamiques urbaines et de leurs interactions avec d'autres dynamiques et variables territoriales. Au fur et à mesure des échanges entre acteurs et del'émergence d'une arène de travail collaboratif, on est ainsi passé d'un modèle démonstrateur à l'échelle communale, définissant les parcelles cadastrales comme entités principales et décrivant le processus d'étalement urbain de manière essentiellement probabiliste, à un modèle plus complexe à l'échelle intercommunale, faisant interagir trois types d'entités spatiales (intercommunalité, pôle urbain et parcelle), couplant des dynamiques d'urbanisation planifiée et d'urbanisation non contrôlée, et décrivant ces dernières à travers deux processus: (i) le mitage agricole influencé par des règles de proximité (réseau routier, zones historiques de mitage...); (ii) le bâti diffus en périphérie des centres urbains (tension foncière).

En termes de légitimité, enfin, la possibilité d'intégrer des perspectives diverses et de les objectiver dans des simulations spatiales a permis de dépasser les clivages politiques «traditionnels» et les visions sectorielles ou purement statistiques et d'amorcer un débat collectif sur le fonctionnement, les effets et la régulation de l'étalement urbain. Les modèles et simulations spatiales s'avèrent ainsi de puissants "objets intermédiaires » (Vinck, 2009) pour travailler à l'interface entre différents mondes sociaux, connecter différents acteurs et, potentiellement, contribuer à remodeler leurs perceptions et interactions (Van Egmond et Zeiss, 2010). Cette dernière propriété doit cependant être considérée avec précaution. En effet, si les représentations cartographiques et dynamiques des impacts de différents scénarios se sont révélées utiles pour retenir l'attention des acteurs et stimuler la discussion, le rendu très réaliste des simulations (à l'échelle de la parcelle cadastrale) a également suscité d'importantes craintes de la part d'un certain nombre de participants, inquiets de voir les résultats mal interprétés ou surinterprétés ou détournés à des fins politiques. Comme nous l'avons décrit plus haut, la perspective et les attentes des acteurs de la filière sucrière concernant le travail de modélisation/simulation et la manière de le présenter illustrent bien ce potentiel.

\section{Conclusion}

Le développement incrémentiel du modèle de simulation de l'étalement urbain a contribué à mettre en place et maintenir une arène organisant les débats autour des évolutions possibles des dynamiques urbaines sur le territoire d'une intercommunalité de La Réunion. Les résultats obtenus montrent qu'un processus d'apprentissage collectif a pu être amorcé, notamment en favorisant des échanges et points de vue sur l'étalement urbain, processus marquant des dynamiques territoriales sur le TCO. Néanmoins, on peut considérer que le cycle d'apprentissage ne s'est pas accompli jusqu'au changement de points de vue des parties et jusqu'à l'émergence d'une convergence de perspectives (Van der Wal et al., 2014). Outre la durée limitée de cet exercice collaboratif, le choix de positionner, dans la démarche, le modèle comme un soutien à la participation en quantifiant et visualisant les effets des scénarios en termes d'occupation du sol, a limité la dimension heuristique de la modélisation. Pour favoriser l'accomplissement du cycle de l'apprentissage collectif, la modélisation devrait pouvoir apporter des connaissances nouvelles sur les impacts territoriaux des scénarios (Albert et al., 2012). Pour boucler l'exercice de prospective, il s'agirait, de mobiliser, en complément, des modèles «experts» qui viseraient à objectiver des impacts liés à des changement d'occupation du sol, comme le ruissellement ou la qualité de l'eau. Ces processus sont en général mal connus et, en conséquence, sources de controverse.

\section{Remerciements}

Ces travaux de recherche ont été en partie financés par l'Agence nationale de la recherche, à travers le projet DESCARTES (ANR 11-AGRO-002-01). 


\section{Bibliographie}

AGORAH (AGENCE POUR L'OBSERVATION DE LA RÉUNION, L'AMÉNAGEMENT ET L'HABITAT) (2008) Densification de la tâche urbaine réunionnaise, tendances et perspectives en 2020. La Réunion, Agence d'urbanisme à la Réunion.

ALBERT, Christian, ZIMMERMANN, Thomas, KNIELING, Jorg et HAAREN, Christina (2012) Social learning can benefit decisionmaking in landscape planning: Gartow case study on climate change adaptation, Elbe valley biosphere reserve. Landscape and Urban Planning, vol. 105, nº 4, p. 347-360.

ALCAMO, Joseph, KOK, Kasper, BUSCH, Gerald, PRIESS, Jorg A., EICKHOUT, Bas, ROUNSEVELL, Mark, ROTHMAN, Dale et HEISTERMANN, Maik (2006) Searching for the future of land: Scenarios from the local to global scale. Dans Éric Lambin et Helmut Geist (dir.) Land-use and landcover change. Springer, Berlin Heidelberg, p. 137-155.

ANTONA, Martine, D'AQUINO, Patrick, AUBERT, Sigrid, BARRETEAU, Olivier, BOISSAU, Stanislas, BOUSQUET, François, DARE, William's, ÉTIENNE, Michel, LE PAGE, Christophe, MATHEVET, Raphael, TREBUIL, Guy et WEBER, Jacques (2005) La modélisation comme outil d'accompagnement. Natures Sciences Sociétés, vol.13, p. 165-168.

BATTON-HUBERT, Mireille, JOLIVEAU, Thierry et LARDON, Sylvie (2008) Modélisation spatiale et décision territoriale participative. Conception et mise en œuvre dans des ateliers chercheursacteurs. Dans Sylvie Lardon et Stéphane Roche (dir.) Représentations spatiales dans les démarches participatives. Revue internationale de géomatique, vol. 18, nº 4 , p. 549-569.
BERTRAND, Nathalie, DOUILLET, AnneCécile et FAURE Alain (2005) L'agriculture périurbaine ou les limites de la campagne à la ville. La construction socio-politique de la question agricole dans la région urbaine grenobloise. Dans Samuel Arlaud et Jean Royou (dir.) Rural-urbain: nouveaux liens, nouvelles frontières. Rennes, Presses Universitaires de Rennes, p. 189-202.

BERTRAND, Nathalie, SOUCHARD, Nadine, ROUSIER, Nicole, MARTIN, Samuel et MICHEELS, Marie-Christine (2006) Quelle contribution de l'agriculture périurbaine à la construction de nouveaux territoires : consensus ou tensions? Revue d'Économie Régionale et Urbaine, vol.3, p. 329-353.

CASH, David W., CLARK, William C., ALCOCK, Franck, DICKSON, Nancy M., ECKLEY, Noelle, GUSTON, David H., JÄGER, Jill et MITCHELL, Ronald B. (2003) Knowledge systems for sustainable development. Proceedings of the National Academy of Sciences, vol. 100, no ${ }^{\circ}$, p. 8086-8091.

CGDD (COMMISSARIAT GÉNÉRAL AU DÉVELOPPEMENT DURABLE - FRANCE) (2012) La revue du CGDD. Urbanisation et consommation de l'espace, une question de mesure. Paris, Ministère de l'Écologie, du Développement durable, du Transport et du Logement.

CRR (CONSEIL RÉGIONAL DE LA RÉUNION) (2011) Projet schéma d'aménagement régional de La Réunion. Rapport vol. 2. La Réunion, Les publications du CRR.

DEAL (DIRECTION DE L'ENVIRONNEMENT, DE L'AMÉNAGEMENT ET DU LOGEMENT - FRANCE) (2012) 20 années de construction neuve à La Réunion, La Réunion, Ministère de l'Environnement, de l'Énergie et de la Mer.

DEGENNE, Pascal, LO SEEN, Danny, PARIGOT, Didier, FORAX, Rémi, TRAN, Annelise, AIT LAHCEN, Ayoub, CURE, Olivier et JEANSOULIN, Robert (2009) Design of a Domain Specific Language for modelling processes in landscapes. Ecological Modelling, vol.220, n²4, p. 3527-3535. 
DEGENNE, Pascal (2012) Une approche générique de la modélisation spatiale et temporelle: application à la modélisation des paysages. Paris, Université Paris-Est, Département de géographie, thèse de doctorat.

GOURMELON, Françoise, HOUET, Thomas, VOIRON-CANICIO, Christine et JOLIVEAU, Thierry (2012) La géoprospective, apport des approches spatiales à la prospective. L'Espace géographique, vol.2, nº41, p. 97-98.

HOUET, Thomas (2015) Usages des modèles spatiaux pour la prospective. Revue internationale de géomatique, 2015, vol. 25, no 1 , p.123-143.

JARRIGE, Françoise, THINON, Pascal et NOUGAREDES, Brigitte (2006) La prise en compte de l'agriculture dans les nouveaux projets de territoires urbains. Exemple d'une recherche en partenariat avec la Communauté d'Agglomération de Montpellier, Revue d'Économie Régionale et Urbaine, vol. 3, p. 393-414.

KOK, Kasper et VERBURG, Peter. H. (2007) Integrated assessment of the land system: The future of land use. Land Use Policy, vol. 24, n³, p. 517-520.

LAMBIN, Éric, GEIST, Helmut et RINDFUSS, Ronald R. (2006) Introduction: Local processes with global impacts. Dans Éric Lambin et Helmut Geist (dir.) Land-use and land-cover change. Springer, Berlin, Heidelberg, p. 1-8.

LARDON, Sylvie et PIVETEAU, Vincent (2005) Méthodologie de diagnostic pour le projet de territoire: une approche par les modèles spatiaux. Géocarrefour, vol. 80, nº 2, p. 75-90.

MAUREL, Pierre, CRAPS, Marc, CERNESSON, Flavie, RAYMOND, Richard, VALKERING, Pieter et FERRAND, Nils (2007) Concepts and methods for analyzing the role of Information and Communication tools (IC-tools) in social learning processes for River Basin Management. Environmental Modelling \& Software, vol. 22, $\mathrm{n}^{\circ} 5$, p. 630-639.
McCARTHY, Michael A. et POSSINGHAM, Hugh P. (2006) Active adaptive management for conservation. Conservation Biology, vol. 21, nº 4 , p. 956-963.

METZGER, Pascale (2004) Les enjeux de la question urbaine dans l'aménagement régional à l'île de La Réunion. Espace populations sociétés, vol.2, p. 361-372.

SAR (SCHÉMAD’AMÉNAGEMENT RÉGIONAL) (2011) Approbation du SAR en conseil d'État. Saint-Denis, Région Réunion.

SOUCHARD, Nadine (2013) Sortie de planification: les voies incertaines de la gouvernance des terres périurbaines. Dans Nathalie Bertrand (dir.) Terres agricoles périurbaines, une gouvernance foncière en construction. Versailles, Éditions Quæ, p. 81-99.

STENSEKE, Marie (2009) Local participation in cultural landscape maintenance: Lessons from Sweden. Land Use Policy, vol. $26, \mathrm{n}^{\circ} 2$, p. 214-223.

TETIS (TERRITOIRE ENVIRONNEMENT TÉLÉDÉTECTION ET INFORMATION SPATIALE) (2016) Ocelet [En ligne]. http://ocelet.teledetection.fr/index. php?tab=home\&lang=fr

VAN BERKEL, Derek B. et VERBURG, Peter H. (2012) Combining exploratory scenarios and participatory backcasting: Using an agent-based model in participatory policy design for a multi-functional landscape. Landscape Ecology, vol. 27, nº 5, p. 641-658.

VAN DER WAL, Merel, DE KRAKER, Joop, OFFERMANS, Astrid, KROEZE, Carolien, KIRSCHNER, Paul A. et ITTERSUM, Martin (2014) Measuring social learning in participatory approaches to natural resource management. Environmental Policy and Governance, vol.24, nº 1, p. 1-15.

VAN EGMOND, Stans et ZEISS, Ragna (2010) Modeling for policy: Science-based models as performative boundary objects for Dutch policy making. Science Studies, vol. $23, \mathrm{n}^{\circ} 1$, p. $58-78$. 
VAN ITTERSUM, Martin K., RABBINGE, Rudy et VAN LATESTEIJN, Henk C. (1998) Land use studies and their role in strategic policy making. Agricultural Systems, vol. 58, n³, p. 309-330.

VERBURG, Peter H., KOK, Kasper, PONTIUS, Robert G. Jr et VELDKAMP, A. (2006) Modeling land-use and land-cover change. Dans Éric Lambin et Helmut Geist (dir.) Land-use and land-cover change. Springer, Berlin Heidelberg, p. 117-135.

VINCK, Dominique (2009) De l'objet intermédiaire à l'objet-frontière. Revue d'anthropologie des connaissances, vol. 3 $\mathrm{n}^{\circ} 1$, p. 51-72.

VOIRON-CANICIO, Christine (2012) L'anticipation du changement en prospective et des changements spatiaux en géoprospective. L'Espace géographique, vol. 2, no 4 , p. 99-110.

VOLK, Martin, LAUTENBACH, Sven, VAN DELDEN, Hedwig, NEWHAM, Lachlan et SEPPELT, Ralf (2010) How can we make progress with decision support systems in landscape and river basin management? Lessons learned from a comparative analysis of four different decision support systems. Environmental Management, vol.46, nº6, p. 834-849.

WALZ, Ariane, LARDELLI, Corina, BEHRENDT, Heiko, GRÊT-REGAMEY, Adrienne, LUNDSTRÖM, Corinne, KYTZIA, Susanne et BEBI, Peter (2007) Participatory scenario analysis for integrated regional modelling. Landscape and Urban Planning, vol.81, nos 1-2, p. 114-131.

WOLLENBERG, Eva, EDMUNDS, David et BUCK, Louise (2000) Using scenarios to make decisions about the future: Anticipatory learning for the adaptive co-management of community forests. Landscape and Urban Planning, vol.47, $\mathrm{n}^{\mathrm{os}} 1-2$, p. 65-77. 